\title{
Smart devices for a smart detection of atrial fibrillation
}

\author{
Juan Benezet-Mazuecos, Camila S. García-Talavera, José Manuel Rubio \\ Department of Cardiology, Hospital Universitario Fundación Jiménez Díaz-Quironsalud, Universidad Autónoma de Madrid, Madrid, Spain \\ Correspondence to: Juan Benezet-Mazuecos, MD, PhD. Unidad de Arritmias, Servicio de Cardiología, Hospital Universitario Fundación Jiménez Díaz- \\ Quironsalud, Avenida Reyes Católicos 2, Madrid 28040, Spain. Email: jbenezet@fjd.es. \\ Provenance: This is an invited Editorial commissioned by the Section Editor Fang-Zhou Liu (Guangdong Cardiovascular Institute, Guangzhou, \\ China). \\ Comment on: Bumgarner JM, Lambert CT, Hussein AA, et al. Smartwatch Algorithm for Automated Detection of Atrial Fibrillation. J Am Coll \\ Cardiol 2018;71:2381-8.
}

Submitted Aug 01, 2018. Accepted for publication Aug 28, 2018.

doi: $10.21037 /$ jtd.2018.08.138

View this article at: http://dx.doi.org/10.21037/jtd.2018.08.138

Atrial fibrillation (AF) is the most frequent sustained cardiac arrhythmia and is associated with significant clinical events and costs. A higher and increasing prevalence of $\mathrm{AF}$ has been reported, mostly due to advances in diagnosis methods (1). AF morbidity and mortality reports are based primarily on symptomatic AF. However, in at least one third of patients, AF is neither clearly symptomatic nor noticeable due to degradation of functional class. This silent form of $\mathrm{AF}$ is incidentally diagnosed during routine physical examinations, pre-operative assessments or population surveys. Regrettably, in some other cases, silent $\mathrm{AF}$ is revealed after complications such as stroke or congestive heart failure have occurred as first manifestation (2). Therefore, lack of symptoms from AF does not mean lack of risk of complications, which are probably present in the same magnitude as in symptomatic AF.

Assessment of the real prevalence of paroxysmal AF is challenging, since the arrhythmia may be brief, completely asymptomatic and difficult to detect. Today's cardiac implantable electronic devices (pacemakers, defibrillators...) can accurately detect asymptomatic cardiac arrhythmias as atrial high rate episodes (AHREs) when the atrial rate exceeds the programmed detection rate during a programmable number of beats (3). The presence of short duration AHREs ( $>5-6$ minutes) has been related to a higher risk of stroke and systemic embolism (4-6). On the other hand, in most of the patients with AHREs and embolic events, the mechanism of stroke may not be related to the AHREs itself. Nevertheless, these episodes seem to be a consistent risk marker for thromboembolic events whatever the ultimate mechanism is (7). AHREs represent a "grey zone" where management recommendations are lacking. Nowadays, the use of anticoagulation in this scenario is unclear in the absence of randomized studies. In any case, it is consistent in all the studies that the longer we see the heart rhythm in patients with AF risk factors the more $\mathrm{AF}$ we detect and these episodes are related to a higher risk for embolic events. In the following years, results from ARTESiA (Apixaban for the Reduction of ThromboEmbolism in Patients With Device-Detected Sub-Clinical Atrial Fibrillation; NCT01938248) and NOAH (NonVitamin K Antagonist Oral Anticoagulants in Patients With Atrial High Rate Episodes; NCT02618577) will provide evidences about the best treatment for this short episodes of AF. Today, management of these patients should be adapted attending to their individual risk factors and $\mathrm{CHA}_{2} \mathrm{DS}_{2} \mathrm{VASc}$ score.

Technological advances in all fields are exciting, particularly in the medical field. New electronic devices (smart phones, smart watches...) can also perform other tasks for us such as assessing our daily steps, walked distance, sleeping hours, calories burned or even tell our heart rate... (8). However, the true expectation for these technological advances is that they may help us to diagnose diseases or detect abnormalities and so we can treat them quickly and prevent complications. This is particularly necessary in AF, a highly prevalent entity with potentially devastating complications. The most important limitation is that $\mathrm{AF}$ usually requires a prolonged monitoring for its diagnosis. On the other hand, smart devices with accurate 


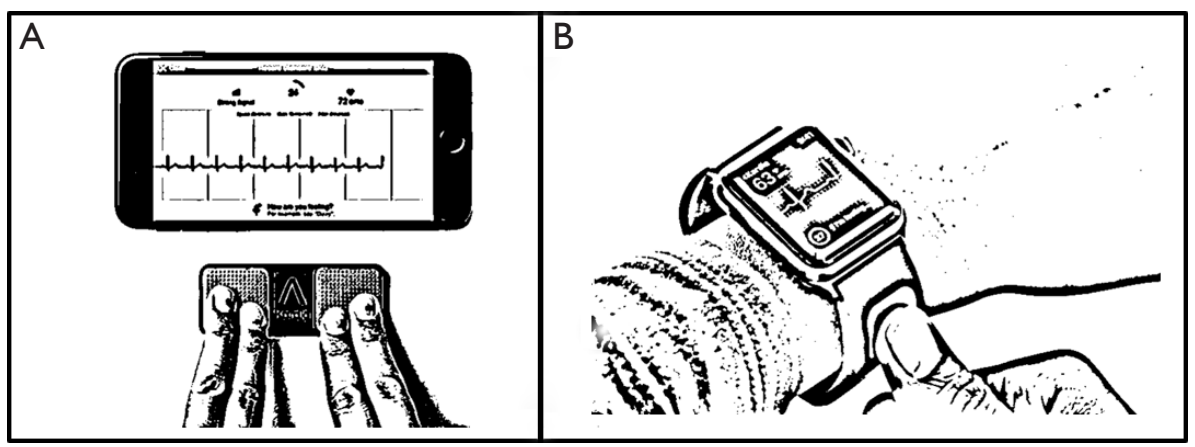

Figure 1 Kardia smart devices for heart rhythm monitoring. (A) Showing the Kardia Mobile consisting in a two electrodes detector for finger tips and a smart phone app that receive the ECG signal (lead I) and it is instantly displayed on the smart phone screen; (B) showing Kardia Band that replaces the original Apple Watch band with an integrated sensor electrode in which placing the thumb a single lead ECG is recorded and displayed on the smart watch screen.

monitoring capabilities might resolve this limitation. Almost every one of us has a cell phone, smart phone or other electronic device that we carry with us every day.

The development of smart phones applications which record a high-quality single lead ECG makes heart rhythm assessment feasible (9) (Figure 1). The Kardia Mobile (Alivecor, Inc., USA) device is a portable smart phone compatible accessory which contains two electrodes. The Kardia Mobile device transmits the signal to the Kardia Mobile app, a downloadable mobile application for smart phones. Recording starts with finger placement of right and left hands on the two electrodes. Finger contact activates ECG recording of bipolar lead I (right hand to left hand) and is transmitted from the device to the phone. The ECG is instantly shown on the smart phone screen. An automated algorithm on the Kardia Mobile app checks the rhythm based on RR wave regularity and it provides a diagnosis citing either "no abnormalities detected", "possible atrial fibrillation" or "this ECG could not be interpreted". It is a FDA-validated algorithm with 97\% sensitivity and 98\% specificity for AF detection. Recently data supports its clinical use. REHEARSE-AF study is a randomized controlled trial for AF detection using an Alivecor Kardia device (10). It included 1,001 patients with CHA2DS2-VASc score $>2$ and non-prior history of AF that were randomized to Kardia monitor or routine care. Nineteen patients in the Kardia group were diagnosed with $\mathrm{AF}$ over the 1-year follow-up versus 5 in the routine care group ( $\mathrm{HR}=3.9 ; \mathrm{P}=0.007)$. Most of the patients were comfortable using the device, finding it easy to use, non-restrictive and not causing anxiety. Kardia Mobile has been also considered a more feasible alternative for prolonged ECG monitoring in cryptogenic stroke patients (11). The MOBILE-AF trial has been designed to evaluate the efficacy of the Kardia Mobile device for AF detection in patients with cryptogenic stroke or transient ischemic attack compared to regular follow-up for AF detection using conventional ECG or Holters. This trial may open new clinical scenarios for this promising technology that is already widely used for the remote detection of $\mathrm{AF}$ and common arrhythmias in routine clinical practice.

During the last years, this technology has been extended to smart watches (Figure 1). The Kardia Band was introduced recently as the first FDA approved Apple Watch accessory that record a rhythm strip equivalent to lead I for 30 seconds, provides an instantaneous and automatic algorithm for rhythm diagnosis of $\mathrm{AF}$ and can transmit this information to the patient's caring physician instantaneously. This technology was tested recently in 100 patients programmed for cardioversion of $\mathrm{AF}$ to prevent unnecessary admissions if the patients have already converted to sinus rhythm (12). Compared to ECG, the Kardia Band interpreted AF with 93\% sensitivity, 84\% specificity. Physician's review of Kardia Band recordings showed 99\% sensitivity, 83\% specificity. Among 113 cases where Kardia Band and physician readings of the same recording were interpretable, agreement was really high (K coefficient 0.88 ). Therefore, the Kardia Band algorithm for AF detection, supported by physician's interpretation, can accurately differentiate AF from sinus rhythm. A recent study en rolling 9,750 Health eHeart Study participants and 51 patients undergoing cardioversion at the University of California, with an Apple Watch who downloaded the 
Cardiogram mobile application, capable of detecting heart rate from the photoplethysmography sensor and pedometer data from the accelerometer, showed that these devices can passively identify AF against the standard practice of ECG recordings among patients at rest when heart rate data are collected at a high temporal resolution (98\% sensitivity and $90 \%$ specificity) (13).

Given the increasing use of smart watches with multitude of downloadable mobile applications, this approach may ultimately be applied for AF screening in large populations. These new devices present important advantages in relation to conventional monitoring techniques. They are easyto-use, noninvasive, safe and can be used on demand. There is not risk of wound infection related to a surgical implantation procedure, does not have to be carried on the body causing discomfort and is cheaper than an implantable cardiac monitor (9). These characteristics are especially interesting when used in children and young patients. A recent study in children showed that smart phone-based event monitor was superior compared with conventional monitoring with a higher diagnostic yield and it was also more acceptable to the patient and parents (14).

But despite the great advantages provided for these new devices, they are not always as smart as supposed and there are some important limitations. Due to the widespread use of these devices in the general population and the large number of free apps some of them without proper certification; it may lead to a great amount of misleading information causing anxiety for arrhythmic events reported by the devices but not professionally confirmed, turning a solution into a problem. It is necessary a learning process to improve the technique of ECG acquisition in the smart phone, which requires a comprehensive education, focused on the patient to refine the obtaining of ECG and thus avoid false positives. It is also important to elaborate recommendations for patients supported by the Professional Cardiology Associations and Societies regarding the appropriate use, interpretation of the events reported and what to do in case of arrhythmia detection. Ongoing studies will also help physicians to provide clinical recommendations in relation to the arrhythmia detected by these devices, especially in AF. Finally, it is estimated that by 2019 annual sales of smart phones and smart watches will reach 50 billion dollars worldwide. Therefore, although more studies and technical improvements are needed, it seems undeniable that this technology will be part of our medical practice and will provide great benefits for our patients.

\section{Acknowledgements}

None.

\section{Footnote}

Conflicts of Interest: The authors have no conflicts of interest to declare.

\section{References}

1. Kannel WB, Abbott RD, Savage DD, et al. Epidemiologic features of chronic atrial fibrillation: the Framingham Study. N Engl J Med 1982;306:1018-22.

2. Savelieva I, Camm AJ. Clinical relevance of silent atrial fibrillation: prevalence, prognosis, quality of life, and management. J Interv Card Electrophysiol 2000;4:369-82.

3. Benezet-Mazuecos J, Rubio JM, Farré J. Atrial high rate episodes in patients with dual-chamber cardiac implantable electronic devices: unmasking silent atrial fibrillation. Pacing Clin Electrophysiol 2014;37:1080-6.

4. Glotzer TV, Hellkamp AS, Zimmerman J, et al. Atrial high rate episodes detected by pacemaker diagnostics predict death and stroke: report of the Atrial Diagnostics Ancillary Study of the MOde Selection Trial (MOST). Circulation 2003;107:1614-9.

5. Glotzer TV, Daoud EG, Wyse DG, et al. The relationship between daily atrial tachyarrhytmia burden from implantable device diagnostics and stroke risk: the TRENDS study. Circ Arrhythm Electrophysiol 2009;2:474-80.

6. Healey JS, Connolly SJ, Gold MR, et al. Subclinical Atrial Fibrillation and the Risk of Stroke. N Engl J Med 2012;366:120-9.

7. Glotzer TV, Ziegler PD. Cryptogenic stroke: Is silent atrial fibrillation the culprit? Heart Rhythm 2015;12:234-41.

8. Mela T. Smartwatches in the Fight Against Atrial Fibrillation: The Little Watch That Could. J Am Coll Cardiol 2018;71:2389-91.

9. Lau JK, Lowres N, Neubeck L, et al. IPhone ECG application for community screening to detect silent atrial fibrillation: A novel technology to prevent stroke. Int J Cardiol 2013;165:193-4.

10. Halcox JP, Wareham K, Cardew A, et al. Assessment of remote heart rhythm sampling using the AliveCor heart monitor to screen for atrial fibrillation the REHEARSEAF study. Circulation 2017;136:1784-94.

11. Treskes RW, Gielen W, Wermer MJ, et al. Mobile phones 
in cryptogenic strOke patients Bringing sIngle Lead

ECGs for Atrial Fibrillation detection (MOBILE-AF):

Study protocol for a randomised controlled trial. Trials 2017;18:402.

12. Bumgarner JM, Lambert CT, Hussein AA, et al.

Smartwatch Algorithm for Automated Detection of Atrial Fibrillation. J Am Coll Cardiol 2018;71:2381-8.

Cite this article as: Benezet-Mazuecos J, García-Talavera CS, Rubio JM. Smart devices for a smart detection of atrial fibrillation. J Thorac Dis 2018;10(Suppl 33):S3824-S3827. doi: $10.21037 /$ jtd.2018.08.138
13. Tison GH, Sanchez JM, Ballinger B, et al. Passive Detection of Atrial Fibrillation Using a Commercially Available Smartwatch. JAMA Cardiol 2018;3:409-16.

14. Macinnes M, Martin N, Fulton H, et al. Comparison of a smartphone-based ECG recording system with a standard cardiac event monitor in the investigation of palpitations in children. Arch Dis Child 2018. [Epub ahead of print]. 